\title{
Radiation Therapy for Locally Recurrent Breast Cancer
}

\author{
Joshua Siglin, Colin E. Champ, Yelena Vakhnenko, \\ Pramila R. Anne, and Nicole L. Simone \\ Department of Radiation Oncology, Kimmel Cancer Center, Jefferson Medical College, Thomas Jefferson University, \\ Philadelphia, PA 19107, USA
}

Correspondence should be addressed to Nicole L. Simone, nicole.simone@jeffersonhospital.org

Received 27 April 2012; Accepted 31 August 2012

Academic Editor: Michael S. Simon

Copyright () 2012 Joshua Siglin et al. This is an open access article distributed under the Creative Commons Attribution License, which permits unrestricted use, distribution, and reproduction in any medium, provided the original work is properly cited.

Approximately one-third of all breast cancer patients experience local recurrence of their tumor after initial treatment. As initial treatment often employs the use of radiation therapy (RT), the standard of care for local breast cancer recurrence after initial breast conserving therapy has traditionally been surgical intervention with mastectomy. However, recent attempts to preserve the intact breast after recurrence with local excision have revealed a potential need for RT in addition to repeat breast conserving surgery as rates of local failure with resection alone remain high. Additionally, local recurrence following initial mastectomy and chest wall RT can be treated with reirradiation to increase local control. Repeating RT, however, in a previously irradiated area, is a complex treatment strategy, as the clinician must carefully balance maximizing treatment effectiveness while minimizing treatment-related toxicity. As a result, physicians have been hesitant to treat recurrent disease with repeat RT with limited data. Results from the current literature are promising and current clinical trials are underway to explore reirradiation modalities which will provide additional information on treatment-related toxicity and outcomes. This paper will review the current literature on repeat radiation therapy for locally recurrent breast cancer.

\section{Introduction}

Of the nearly 300,000 cases of invasive and in situ breast cancers diagnosed in 2010 alone, $20-40 \%$ of these patients will experience a local recurrence (LR) and approximately half of those will have isolated recurrences [1]. Optimal local control is noted to be important for patient outcomes. Local recurrences still occur at rates reported in large, randomized trials between 3-20\% rate for patients receiving breast conserving therapy (BCT) and a $2-12 \%$ for patients who have undergone mastectomy [2-4]. Accordingly, treatment of locally recurrent breast cancer remains important in the care of breast cancer patients. Patient and tumor characteristics, as well as initial treatment, have a significant impact on the treatment options offered for recurrent disease. Given the heterogeneous nature of local breast cancer recurrence, several treatment options are available and in this paper we will further outline the role of radiation therapy (RT) which is one such treatment modality.

\section{Recurrence in Patients Treated Initially with BCT}

Mastectomy remains the standard of care for patients with in-breast tumor recurrence (IBTR) after initial treatment with BCT. Mastectomy in the setting of recurrent disease results in a second local recurrence rate of $2-31 \%$ [5]. In patients with an in-breast tumor recurrence (IBTR), fiveyear overall survival rates after mastectomy range from 52 to $84 \%$ in various series [6-10]. While mastectomy remains the current standard of care for IBTR, a growing body of literature describes repeat BCT in the setting of locally recurrent disease.

2.1. Repeat Breast Conserving Therapy without Reirradiation. More women are choosing to preserve their breast in the setting of recurrence with repeat BCT. Initial experiences with BCT for recurrence focused on partial mastectomy alone without repeat irradiation. Kurtz et al. reported on 52 
TABLE 1: Outcomes of patients treated with breast conserving surgery alone following in-breast local recurrence.

\begin{tabular}{lcccc}
\hline Study & Number of patients & Median followup (months) & Local recurrence (\%) & 5-year overall survival (\%) \\
\hline Kurtz et al. [11] & 55 & 51 & 32 & NR \\
Abner et al. [12] & 16 & 39 & 31 & 81 \\
Salvadori et al. [6] & 57 & 73 & 19 & 85 \\
Voogd et al. [13] & 16 & 52 & 38 & NR \\
Ishitobi et al. [14] & 78 & 40 & 21.2 & NR \\
\hline
\end{tabular}

NR: not reported.

patients who had received wide local excision for isolated LR. Patients with mobile tumors less than $2 \mathrm{~cm}$ in diameter and no signs of rapid progression were carefully selected for breast preservation. In this group, the five-year local control and cause-specific survival were $79 \%$ which are comparable to results noted with mastectomy. Interestingly, it was noted that local control was doubled when negative margins were achieved with local excision on second surgery. Therefore, it seems that the potential exists to avoid a mastectomy in the setting of recurrent disease if adequate margins are obtained [11].

This was explored further by Salvadori et al. in a large series reporting on patients receiving a mastectomy or a local resection for isolated LR. With a median followup of 6 years, the risk of a second local recurrence was significantly increased in the local resection group at $19 \%$ versus $4 \%$ for total mastectomy [6]. These results were echoed by Abner et al. and Voogd et al., showing a LR rate of $31 \%$ at 3.5 years and $38 \%$ at 4.5 years, respectively, with a local resection alone (Table 1) [12-14].

While these rates of recurrence are significantly greater than those from total mastectomy, they are comparable to the $35 \%$ risk of recurrence found in upfront breast conserving surgery without the addition of RT. This has led investigation into the feasibility of repeat irradiation to the IBTR recurrence after resection to minimize the risk of local recurrence. To date, repeat radiation has been described using various treatment techniques such as external beam radiation therapy (EBRT), intraoperative radiation therapy (IORT), and brachytherapy.

\section{Second Breast Conserving Therapy with Repeat Radiation Therapy}

Overall, the published data on reirradiation have shown promising results. In terms of local control, reported rates range from $77-100 \%$, with the limited available prospective data revealing a very favorable $7-11 \%$ rate of second LR. Furthermore, acceptable rates of toxicity and cosmesis have been obtained in all reported series, regardless of variations in RT technique for repeat partial breast irradiation. The methodologies previously utilized in the literature will be reviewed, along with the associated outcomes data.

3.1. External Beam Radiation Therapy. As early as the mid1980s, EBRT was employed to reirradiate patients with breast cancer recurrence. Deutsch has reported on approximately
40 women with IBTR following partial mastectomy and radiation therapy. Initial RT consisted of $50 \mathrm{~Gy}$ to the whole breast in 2 Gy fractions with or without tumor bed boost. At the time of recurrence, a median of 63 months from initial treatment, partial breast reirradiation $(\mathrm{PBrI})$ to a dose of $50 \mathrm{~Gy}$ in $2 \mathrm{~Gy}$ daily fractions was delivered to the entire operative quadrant using electrons of appropriate energy. Deutsch showed that among 39 patients at a median followup of 52 months, $77 \%$ of patients had an intact breast free of tumor. Treatment was well tolerated but alteration in skin pigmentation was reported as well as $25 \%$ of evaluated patients having fair to poor cosmesis [15].

A heterogeneous group of 29 patients receiving reirradiation with $3 \mathrm{D}$-conformal RT were reviewed by Würschmidt et al., of which 4 received second breast conserving therapy. Cosmetic results were good to excellent with no Grade 3 or higher toxicity in this small group of patients [16].

The Radiation Therapy Oncology Group (RTOG) is evaluating a similar treatment approach with external beam partial breast radiation therapy in its current phase II trial. The radiation volume will consist of the surgical margin plus CTV and PTV expansions to generate a $2.5 \mathrm{~cm}$ margin excluding the skin surface. This will result in a significantly smaller treatment volume (Figure 1) than the initial RT volume, which usually encompasses the entire breast. Twice daily fractions of $1.5 \mathrm{~Gy}$ will be administered to a total dose of $45 \mathrm{~Gy}$.

3.2. Intraoperative Radiation Therapy. Single institution data on IORT utilized the Intrabeam device, with a median applicator size of $4.0 \mathrm{~cm}$ (Carl Zeiss, Oberkochen, Germany), which delivered a median dose of $20 \mathrm{~Gy}$ to the applicator surface. In this study, postprocedure margins were noted to range from 1 to $10 \mathrm{~mm}$ and after more than 2 years of followup no local recurrences were noted. IORT was also well tolerated with no Grade 3 or higher toxicity and a majority of excellent or good cosmetic outcomes [17].

3.3. Interstitial Brachytherapy. The use of interstitial brachytherapy as a method of delivering focal radiation in the setting of locally recurrent breast cancer has been well documented. A French retrospective study of almost 70 patients who underwent repeat lumpectomy followed by interstitial brachytherapy revealed promising 5-year OS and freedom from second local recurrence (FFLR2). Despite the dissimilar treatment modality, Hannoun-Levi et al. showed results similar to those of Deutsch. A 77\% rate of freedom 

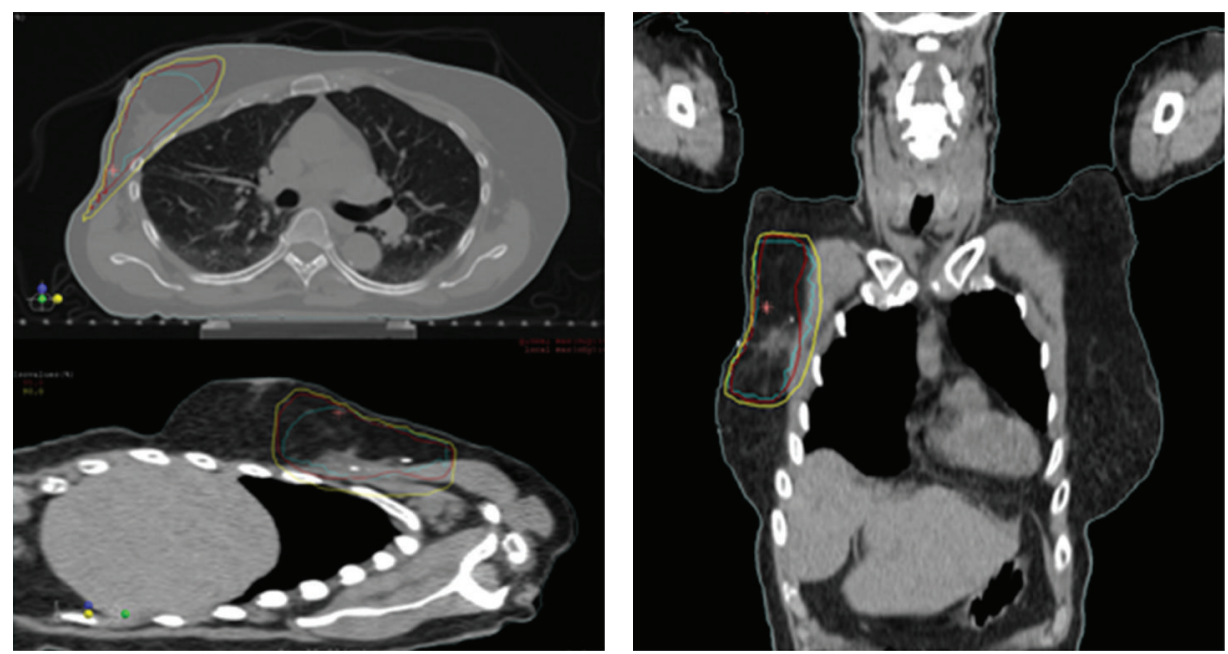

Figure 1: Partial breast radiation therapy plan utilizing external beam radiation therapy with photons. Blue: planning target volumeevaluation (PTVeval), red: 95\% isodose line, yellow: 90\% isodose line.

from second local recurrence was noted in 70 patients. Interestingly, in this group of patients treated with interstitial brachytherapy, multivariate analysis revealed the subset of patients treated with 5 or more wires had a markedly improved FFLR2 of nearly $95 \%$ at 5 years. This treatment course delivered a median of 7.5 years from initial diagnosis and was also well tolerated with minimal acute toxicity. A $10 \%$ rate of late Grade 3 toxicity was noted, most commonly fibrosis [18]. While only in abstract form, a much larger retrospective series of 217 patients receiving second conservative treatment with lumpectomy and interstitial brachytherapy was reported on at the World Congress of Brachytherapy in 2012. The 5-year local recurrence rate was a promising $5.6 \%$ with actuarial OS at 5 years reported as $88.7 \%$. Furthermore, cosmesis was good to excellent in $85 \%$ of patients with an $11 \%$ rate of Grade 3 and 4 toxicity noted [19].

While the aforementioned retrospective data provides insight on the safety and efficacy of PBrI, limited prospective data exist for reirradiation in the setting of locally recurrent breast cancer. Three prospective trials have reported encouraging local control rates, with all studies employing brachytherapy techniques to deliver a second course of radiation therapy. A phase II trial from Austria enrolled 39 patients with IBTR who refused mastectomy and opted for a second breast conserving treatment. Similarly, Guix et al. conducted a study of a second breast conserving treatment in 36 women with limited-size, low-risk local recurrence occurring at a greater than 12 month interval who had refused mastectomy. Patients were excluded from both studies if LR was within 12 months of initial treatment. There was a median interval between treatments of nearly 11 years in the study by Kauer-Dorner et al., but only 3.2 years in the Guix et al. study.

Radiation therapy techniques also differed in these two trials. The Austrian group utilized multicatheter interstitial pulsed dose rate (PDR) brachytherapy following an R0 repeat resection. Mean physical, total dose of $50.1 \mathrm{~Gy}$ in fractions of 0.6 to $1 \mathrm{~Gy}$ was prescribed. Of note, 10 patients had received chemotherapy and 24 received hormonal therapy. The Spanish group employed an HDR technique to deliver $30 \mathrm{~Gy}$ in 12 fractions over a period of 5 days. Adjuvant chemotherapy was given to 13 patients, while 8 patients received hormonal therapy $[20,21]$.

Additionally, a smaller phase I/II study utilized low dose rate brachytherapy, initially to a total dose of $30 \mathrm{~Gy}$ in 6 patients. The dose was escalated to $45 \mathrm{~Gy}$ for the subsequent 9 patients as toxicity was minimal. Consistent with the majority of data, an admixture of systemic therapy was reported with a portion of patients receiving chemotherapy or hormonal therapy [22].

In this phase I/II trial, Chadha et al. reported an $89 \%$ local control rate, but patient numbers were low and median followup was only 36 months. However, similar 5-year actuarial local control of $93 \%$ was seen in the larger Austrian study with a median followup of 57 months. In this study, 5year OS and DFS were $87 \%$ and $77 \%$, respectively. Ten-year actuarial data were reported in the Spanish study, showing an OS of $96.7 \%$, DFS of $64.4 \%$, and local control of $89.4 \%$.

Guix et al. reported low treatment-related toxicity, with no Grade 3 or 4 events reported. Only $4 \%$ of patients in this study had unsatisfactory cosmetic outcomes. Kauer-Dorner et al. did note Grade 3 breast tissue fibrosis in 1 woman and 3 women had Grade 3 pain. Poor or unacceptable cosmesis was documented in 6 patients by independent observers; however, only 2 patients self-reported this level of cosmetic outcome. Thus equivalent local tumor control to mastectomy was obtained by second BCT with good cosmesis and moderate morbidity. Overall, the addition of PBrI to repeat breast conserving surgery appears to be both efficacious and well tolerated (Table 2). However, when considering the use of a second breast conserving therapy it is important to remember that the study populations were highly selected patients with small local recurrences occurring more than one year from initial treatment [2022]. 


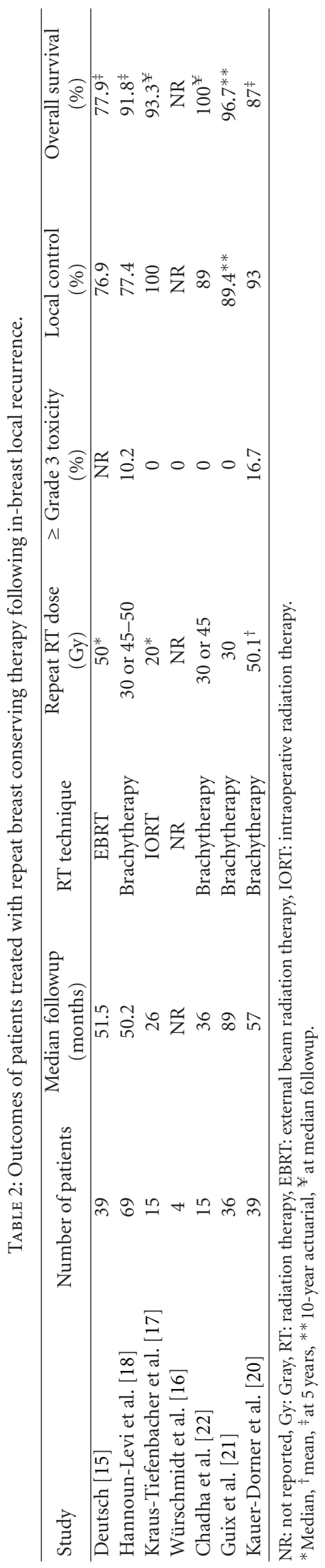


3.4. Future Directions. Further study of repeat BCT employing surgery and reirradiation with partial breast is currently underway in a phase II trial, RTOG 1014. This study will evaluate the adverse events associated with the use of PBrI in patients with recurrent breast cancer following a marginnegative repeat lumpectomy. As in the Austrian study, recurrence within 12 months is an exclusion criterion. External beam radiation therapy is being administered to a total dose of $45 \mathrm{~Gy}$ using a $1.5 \mathrm{~Gy}$ twice daily fractionation. The study will secondarily evaluate endpoints of local control, freedom from mastectomy, cosmesis and OS.

\section{Recurrence in Patients Who Received Initial Mastectomy}

Guidelines for the treatment of local recurrence in patients treated initially with mastectomy are slightly more complex than for those patients who received upfront BCT. The standard of care is currently surgical resection, if technically feasible. Similar to patients who had prior BCT, systemic therapy is to be considered. In patients who have not previously received RT, the NCCN Guidelines recommend chest wall and nodal irradiation. There is a large body of literature demonstrating the use of radiation therapy for chest wall recurrence, including data on chest wall reirradiation. However, caution is warranted in repeat chest wall RT as aggregate doses of $100 \mathrm{~Gy}$ or higher can pose significant risk of toxicity such as skin ulceration, brachial plexopathy, osteonecrosis, rib fracture, and cardiomyopathy.

4.1. Chest Wall Radiation Therapy without Prior RT. In the context of patients who had not had prior radiation, the addition of chest wall radiation therapy at the time of recurrence has well-documented utility for improvement of local control [23, 24]. Halverson et al. reported the Washington University experience with chest wall radiation therapy for local-regional recurrence following mastectomy [25]. Patients treated to a large RT field encompassing the entire chest wall, FFR, were $75 \%$ and $63 \%$ at 5 and 10 years, compared with $36 \%$ and $18 \%$ in patients treated to a smaller field. In this study, field size proved to be an important factor for freedom from recurrence (FFR) and it was recommended that the entire chest wall is treated. A radiation dose of $60 \mathrm{~Gy}$ seemed to adequately control tumors less than $3 \mathrm{~cm}$ in this study, while only half of larger tumors were controlled by doses of up to $70 \mathrm{~Gy}$.

Another single institution retrospective review [26] published by Hsi et al. treated patients at the Hospital of the University of Pennsylvania with a chest wall recurrence following mastectomy using radiation to a median chest wall dose of $50 \mathrm{~Gy}$ along with $10 \mathrm{~Gy}$ scar boost. At a median followup of 8.4 years, the ten-year actuarial local control was $86 \%$, overall survival $72 \%$, and cause specific survival $77 \%$ [27]. It was found that patients who had greater than 2-year disease-free interval after mastectomy, isolated chest wall recurrence, a tumor less than $3 \mathrm{~cm}$, or a completely resected tumor, seemed to respond most favorable to radiation.
4.2. Repeat Chest Wall Radiation Therapy. While the addition of chest wall RT for patients with local recurrence in patients without previous RT is fairly straightforward, repeat irradiation is more controversial due to the high cumulative doses that would be achieved and potential toxicity. Local control with repeat surgery alone remains unacceptable with 5-year local control rates of only $33 \%$ [28]. Subsequently, the use of repeat chest wall RT has been explored for a second curative intent treatment despite the concerns over possible toxicity.

A multi-institutional study by Wahl et al. reviewed the feasibility of chest wall reirradiation. Eighty-one patients received an initial median dose of $60 \mathrm{~Gy}$ and after local recurrence underwent a second course of RT to the chest wall. Median dose for the second treatment course was $48 \mathrm{~Gy}$. Two-thirds of patients were free of local disease at 12 months, with the local DFS rate significantly higher in patients without gross disease. Overall, the complete response rate was 57\% [29]. Three Grade 3 late toxicities occurred, with patients experiencing fibrosis, infection, and lymphedema. The lone Grade 4 event, dermatitis, occurred in a patient who had a cumulative RT dose of $\geq 120 \mathrm{~Gy}$.

A German retrospective study from the University of Tübingen was recently published by Muller et al. reviewing data on the role of repeat surgery and chest wall reirradiation for curative intent. Forty-two women had received an initial median dose of $54 \mathrm{~Gy}$ and after local recurrences received either surgery followed by chest wall reirradiation or chest wall reirradiation alone. The second course of radiation therapy was conventionally fractionated to a median dose of $60 \mathrm{~Gy}$. The median interval between courses of RT was slightly longer than that reported by Wahl et al., 53 months versus 38 months. At a median followup of 41 months, the estimated 5-year local control was $62 \%$ and 5 -year OS was $59 \%$. Only 2 cases of acute Grade 3 skin toxicity were noted. Eight cases of late Grade 3 skin toxicity were reported and no acute or late Grade 4 toxicity occurred [30].

These studies indicate that chest wall reirradiation can provide an improved prognosis for women with local recurrence and can be administered with acceptable acute and late toxicities following initial treatment of breast cancer with surgery and postoperative radiation therapy.

\section{Conclusion}

Despite a growing body of literature, the role of reirradiation for locally recurrent breast cancer remains ill-defined. Repeat breast conserving therapy has shown promise in retrospective reviews and small prospective studies. The role of PBrI will hopefully be further elucidated by more prospective studies, including the ongoing RTOG trial. In patients who have previously received chest wall RT following mastectomy, treatment of local recurrence with reirradiation has been shown to be feasible. Given the heterogeneity in the recurrent breast cancer patient population, reirradiation remains an individualized treatment decision.

\section{Conflict of Interests}

The authors report no conflict of interests. 


\section{Acknowledgment}

This research was supported in part by the Kimmel Cancer Center's NCI Cancer Center Support Grant P30 CA56036.

\section{References}

[1] B. Gerber, M. Freund, and T. Reimer, "Recurrent breast cancer: treatment strategies for maintaining and prolonging good quality of life," Deutsches Arzteblatt, vol. 107, no. 6, pp. 85-91, 2010.

[2] B. Fisher, S. Anderson, J. Bryant et al., "Twenty-year follow-up of a randomized trial comparing total mastectomy, lumpectomy, and lumpectomy plus irradiation for the treatment of invasive breast cancer," The New England Journal of Medicine, vol. 347, no. 16, pp. 1233-1241, 2002.

[3] U. Veronesi, N. Cascinelli, L. Mariani et al., "Twentyyear follow-up of a randomized study comparing breastconserving surgery with radical mastectomy for early breast cancer," The New England Journal of Medicine, vol. 347, no. 16, pp. 1227-1232, 2002.

[4] J. A. Van Dongen, A. C. Voogd, I. S. Fentiman et al., "Long-term results of a randomized trial comparing breastconserving therapy with mastectomy: European organization for research and treatment of cancer 10801 trial," Journal of the National Cancer Institute, vol. 92, no. 14, pp. 1143-1150, 2000.

[5] H. M. Kuerer, D. W. Arthur, and B. G. Haffty, "Repeat breast-conserving surgery for in-breast local breast carcinoma recurrence: the potential role of partial breast irradiation," Cancer, vol. 100, no. 11, pp. 2269-2280, 2004.

[6] B. Salvadori, E. Marubini, R. Miceli et al., "Reoperation for locally recurrent breast cancer in patients previously treated with conservative surgery," British Journal of Surgery, vol. 86, no. 1, pp. 84-87, 1999.

[7] K. Dalberg, A. Mattsson, K. Sandelin, and L. E. Rutqvist, "Outcome of treatment for ipsilateral breast tumor recurrence in early-stage breast cancer," Breast Cancer Research and Treatment, vol. 49, no. 1, pp. 69-78, 1998.

[8] C. C. Cajucom, T. N. Tsangaris, T. Nemoto, D. Driscoll, R. B. Penetrante, and E. D. Holyoke, "Results of salvage mastectomy for local recurrence after breast- conserving surgery without radiation therapy," Cancer, vol. 71, no. 5, pp. 1774-1779, 1993.

[9] A. Recht, S. J. Schnitt, J. L. Connolly et al., "Prognosis following local or regional recurrence after conservative surgery and radiotherapy for early stage breast carcinoma," International Journal of Radiation Oncology Biology Physics, vol. 16, no. 1, pp. 3-9, 1989.

[10] M. P. Osborne, P. I. Borgen, G. Y. Wong, P. P. Rosen, and B. McCormick, "Salvage mastectomy for local and regional recurrence after breast-conserving operation and radiation therapy," Surgery Gynecology and Obstetrics, vol. 174, no. 3, pp. 189-194, 1992.

[11] J. M. Kurtz, J. Jacquemier, J. Torhorst et al., "Conservation therapy for breast cancers other than infiltrating ductal carcinoma," Cancer, vol. 63, no. 8, pp. 1630-1635, 1989.

[12] A. L. Abner, A. Recht, T. Eberlein et al., "Prognosis following salvage mastectomy for recurrence in the breast after conservative surgery and radiation therapy for early-stage breast cancer," Journal of Clinical Oncology, vol. 11, no. 1, pp. 44-48, 1993.

[13] A. C. Voogd, G. van Tienhoven, H. L. Peterse et al., "Local recurrence after breast conservation therapy for early stage breast carcinoma: detection, treatment, and outcome in 266 patients. Dutch study group on Local Recurrence after Breast Conservation (BORST)," Cancer, vol. 85, no. 2, pp. 437-446, 1999.

[14] M. Ishitobi, Y. Komoike, S. Nakahara, K. Motomura, H. Koyama, and H. Inaji, "Repeat lumpectomy for ipsilateral breast tumor recurrence after breast-conserving treatment," Oncology, vol. 81, pp. 5381-5366, 2011.

[15] M. Deutsch, "Repeat high-dose external beam irradiation for in-breast tumor recurrence after previous lumpectomy and whole breast irradiation," International Journal of Radiation Oncology Biology Physics, vol. 53, no. 3, pp. 687-691, 2002.

[16] F. Würschmidt, J. Dahle, C. Petersen, C. Wenzel, M. Kretschmer, and C. Bastian, "Reirradiation of recurrent breast cancer with and without concurrent chemotherapy," Radiation Oncology, vol. 3, no. 1, article 28, 2008.

[17] U. Kraus-Tiefenbacher, L. Bauer, A. Scheda et al., "Intraoperative radiotherapy (IORT) is an option for patients with localized breast recurrences after previous external-beam radiotherapy," BMC Cancer, vol. 7, article 178, 2007.

[18] J. M. Hannoun-Levi, G. Houvenaeghel, S. Ellis et al., "Partial breast irradiation as second conservative treatment for local breast cancer recurrence," International Journal of Radiation Oncology Biology Physics, vol. 60, no. 5, pp. 1385-1392, 2004.

[19] J. Hannoun-Levi, D. Kauer-Dorner, J. Gal et al., "138 second conservative treatment for ipsilateral breast cancer recurrence: GEC-ESTRO Breast WG study," Radiotherapy and Oncology, vol. 103, supplement 2, p. S56, 2012.

[20] D. Kauer-Dorner, R. Potter, A. Resch et al., "Partial breast irradiation for locally recurrent breast cancer within a second breast conserving treatment: alternative to mastectomy? Results from a prospective trial," Radiotherapy and Oncology, vol. 102, no. 1, pp. 96-101, 2012.

[21] B. Guix, J. A. Lejárcegui, J. I. Tello et al., "Exeresis and brachytherapy as salvage treatment for local recurrence after conservative treatment for breast cancer: results of a tenyear pilot study," International Journal of Radiation Oncology Biology Physics, vol. 78, no. 3, pp. 804-810, 2010.

[22] M. Chadha, M. Trombetta, S. Boolbol, and M. P. Osborne, "Managing a small recurrence in the previously irradiated breast. Is there a second chance for breast conservation?" Oncology, vol. 23, no. 11, pp. 933-940, 2009.

[23] A. Schuck, S. Könemann, B. Matthees et al., "Radiotherapy in the treatment of locoregional relapses of breast cancer," British Journal of Radiology, vol. 75, no. 896, pp. 663-669, 2002.

[24] B. Stadler and H. D. Kogelnik, "Local control and outcome of patients irradiated for isolated chest wall recurrences of breast cancer," Radiotherapy and Oncology, vol. 8, no. 2, pp. 105-111, 1987.

[25] K. J. Halverson, C. A. Perez, R. R. Kuske, D. M. Garcia, J. R. Simpson, and B. Fineberg, "Isolated local-regional recurrence of breast cancer following mastectomy: radiotherapeutic management," International Journal of Radiation Oncology Biology Physics, vol. 19, no. 4, pp. 851-858, 1990.

[26] F. Schwaibold, B. L. Fowble, L. J. Solin, D. J. Schultz, and R. L. Goodman, "The results of radiation therapy for isolated local regional recurrence after mastectomy," International Journal of Radiation Oncology Biology Physics, vol. 21, no. 2, pp. 299-310, 1991.

[27] R. A. Hsi, A. Antell, D. J. Schultz, and L. J. Solin, "Radiation therapy for chest wall recurrence of breast cancer after mastectomy in a favorable subgroup of patients," International Journal of Radiation Oncology Biology Physics, vol. 42, no. 3, pp. 495-499, 1998. 
[28] K. K. Dahlstrom, A. P. Andersson, M. Andersen, and C. Krag, "Wide local excision of recurrent breast cancer in the thoracic wall," Cancer, vol. 72, no. 3, pp. 774-777, 1993.

[29] A. O. Wahl, A. Rademaker, K. D. Kiel et al., "Multiinstitutional review of repeat irradiation of chest wall and breast for recurrent breast cancer," International Journal of Radiation Oncology Biology Physics, vol. 70, no. 2, pp. 477-484, 2008.

[30] A. C. Muller, F. Eckert, V. Heinrich, M. Bamberg, S. Brucker, and T. Hehr, "Re-surgery and chest wall re-irradiation for recurrent breast cancer: a second curative approach," BMC Cancer, vol. 11, article 197, 2011. 


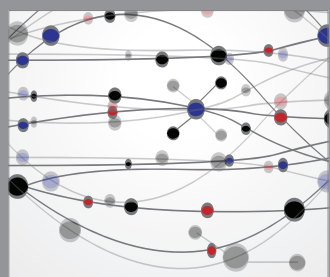

The Scientific World Journal
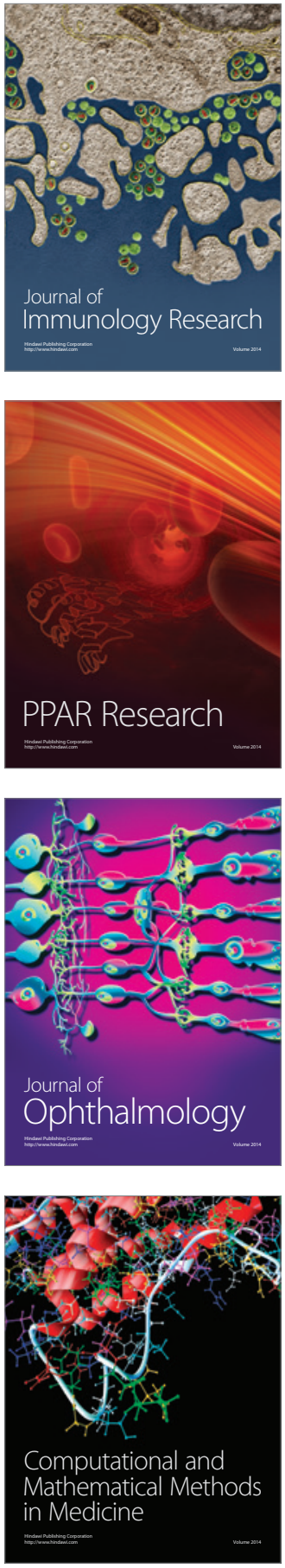

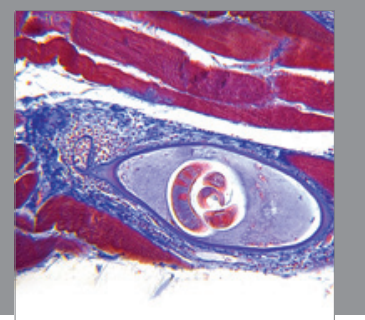

Gastroenterology

Research and Practice
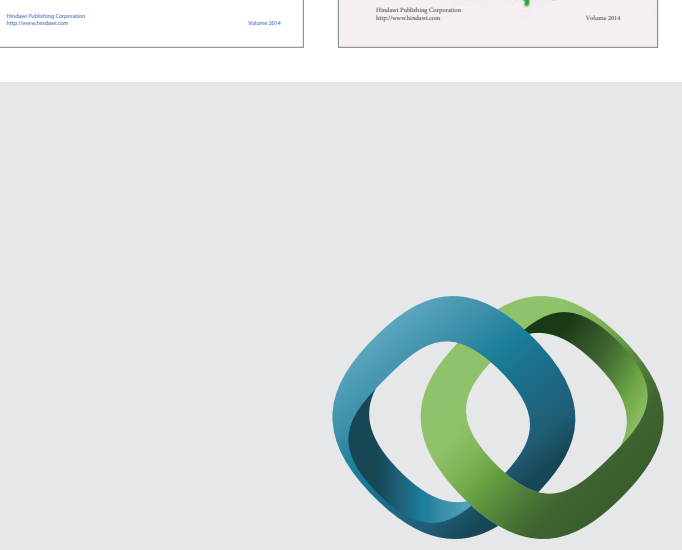

\section{Hindawi}

Submit your manuscripts at

http://www.hindawi.com
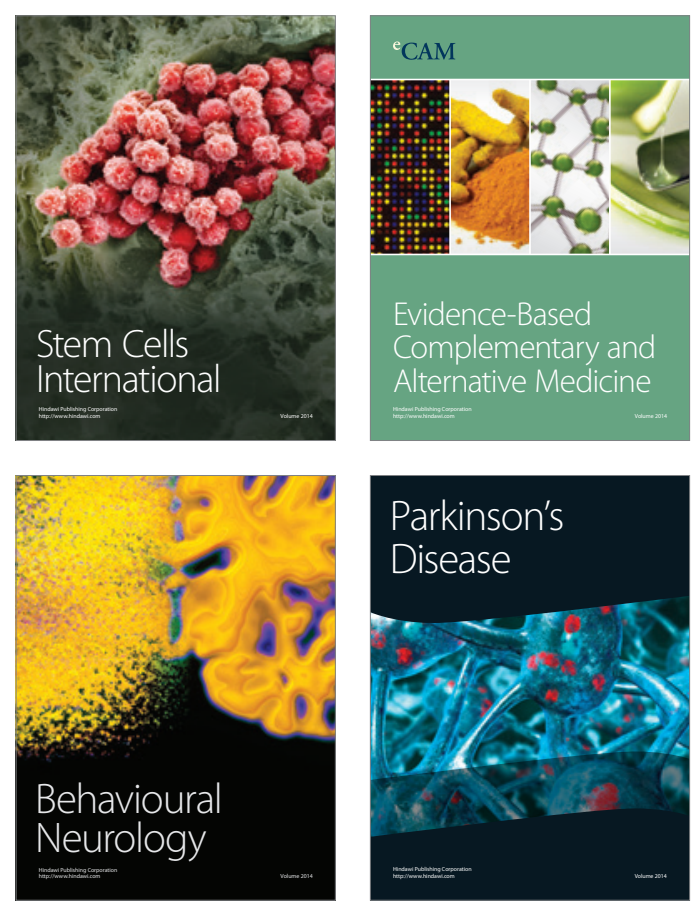

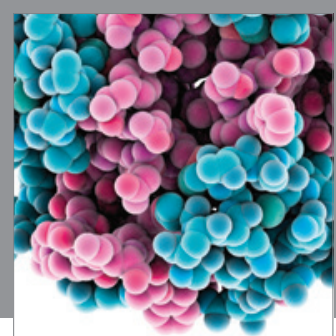

Journal of
Diabetes Research

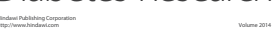

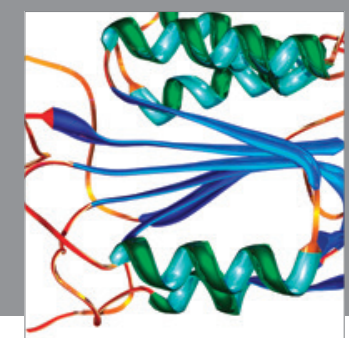

Disease Markers
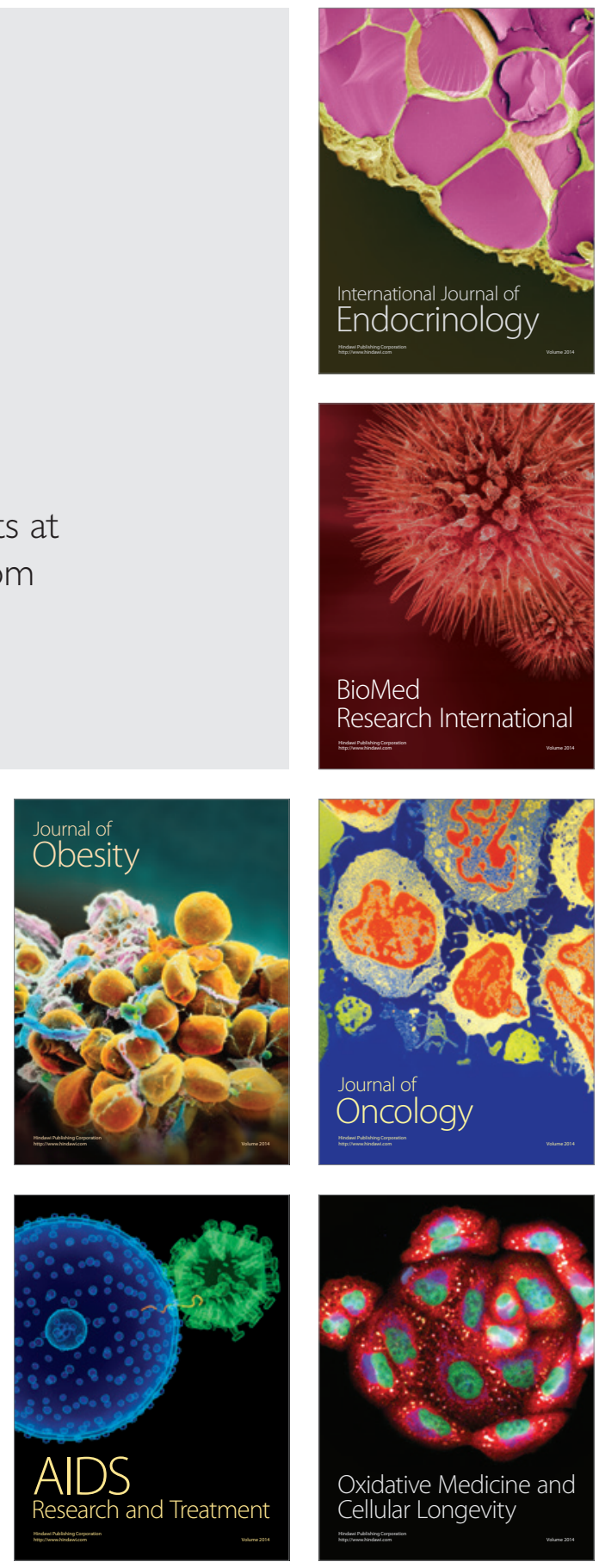